\title{
Educación médica y de ciencias de la salud basada en evidencia: perspectivas y desafíos
}

\author{
Evidence Based Medical Education and Health Sciences: Perspectives and Challenges \\ Carolina Guerra Ferrada', Paola Carrasco Aldunate ${ }^{2}$
}

\begin{abstract}
Resumen
La educación médica basada en evidencia ha proporcionado los estándares para fundamentar la práctica docente. En la medida que los profesores tomen decisiones educativas basadas en evidencia, se lograrán mejores resultados de aprendizaje en los estudiantes y esto se traducirá en que los nuevos profesionales, brinden una atención de salud de calidad a las personas. También es importante incorporar en este proceso de toma de decisiones basados en evidencia, a las ciencias de la salud, ya que este hecho favorecerá el trabajo colaborativo e interdisciplinar, por consiguiente, se favorecerá la salud de la comunidad. El propósito de este artículo es analizar las perspectivas de la educación médica y de las ciencias de la salud y plantear los desafíos actuales y futuros de esta.
\end{abstract}

Palabras clave: educación médica basada en evidencia; educación de ciencias de la salud; educación médica; investigación en educación médica.

\begin{abstract}
Evidence based medical education has provided the standards to inform teaching practice. To the extent that teachers make educational decisions based on evidence, better learning results will be achieved in students and this will translate into new professionals providing quality health care to people. It is also important to incorporate health sciences into this evidence-based decision-making process, since this fact will favor collaborative and interdisciplinary work, therefore, the health of the community will be favored. The purpose of this article is to analyze the perspectives of medical education and the health sciences and to present the current and future challenges of this.
\end{abstract}

Keywords: evidence based medical education; health sciences education; medical education; medical education research

Fecha de envío: 2021-10-26- Fecha de aceptación: 2021-12-10

\section{Introducción}

En la década de los noventa se inicia el proceso de transformación de la educación tradicional de los médicos. Harden, R. et al. (1984), incorpora un nuevo paradigma educativo en la formación de profesionales médicos. Este enfoque pone en el centro al estudiante como el protagonista de su aprendizaje, lo convierte en un ente activo y el profesor se transforma en un guía (Harden et al., 1984). Junto con este cambio, los profesionales comienzan a analizar el sustento que existe detrás de la práctica docente, ya que, a luz de este nuevo modelo, se necesita fundamentar con "evidencia científica" la toma de decisiones educativas (Harden \& Lilley, 2000). En este sentido, Van der Vleuten et al. (2000) mencionan que "los médicos están entrenados para tomar decisiones basadas en evidencia, pero cuando asumen su rol docente parecen abandonar todo su enfoque crítico sobre qué funciona y qué no funciona, y basarse en la tradición y la intuición". Además, al comparar el rol asistencial y de investigación que tienen los médicos con el rol docente, este último no tiene una estructura tan precisa, como las otras áreas (Rivera \& Letelier, 2007). Este punto no es menor, debido a que cada médico debe formar estudiantes que brinden una atención con altos estándares de calidad y seguridad, sin embargo, no todos tienen una formación definida en docencia e incluso pueden ser expertos en sus respectivas áreas de interés y no necesariamente ser buenos docentes (Petersen, 1999; Sánchez-Mendiola, 2012; Norman, 2000). En Latinoamérica y en otras profesiones no médicas, el desarrollo de la "evidencia" como referente es posterior,

(1) Departamento de Salud del Adulto y Senescente, Escuela de Enfermería, Pontificia Universidad Católica de Chile.

Autor de correspondencia: ccguerra@uc.cl 
por ende, si bien en profesiones como Enfermería y Odontología, existe un progreso que casi puede llegar al nivel médico, no es así en otras profesiones tales como, kinesiología, fonoaudiología, nutrición, donde es más incipiente (Cobos, 2016; Vidal \& Vicedo, 2017). Por consiguiente, este artículo tiene por objetivo analizar las perspectivas de la educación médica y de las ciencias de la salud y plantear los desafíos actuales y futuros de esta.

\section{Perspectivas de la educación médica}

En agosto del año 1999, se lleva a cabo el congreso de la "Association for Medical Education in Europe" (AMEE), donde se discute la importancia de sustentar con evidencia la "experiencia" del profesor, ya que hasta ese momento la "intuición" era el gran argumento que sostenía la práctica docente (Harden et al., 1984; Hart \& Harden, 2000; Dauphinee \& Wood-Dauphinee, 2004; Sánchez-Mendiola, 2012;). Es así como, Harden \& Hart (2000), crean la colaboración "Best Evidence Medical Education" (BEME) que corresponde a "un grupo internacional de personas e instituciones comprometidas con la promoción de políticas y prácticas basadas en evidencia en la educación de los profesionales de la salud" (Thistlethwaite \& Hammick, 2010). El nacimiento de la colaboración BEME ordena y fundamenta la toma de decisiones en educación, debido a que, por una parte, respalda con literatura científica la actividad docente y por otra, no elimina la "experiencia" del profesor, sino más bien la integra en esta nueva mirada (Harden \& Lilley, 2000). En este sentido, Harden \& Lilley (2000) proponen una estructura similar a la dispuesta en Medicina Basada en la Evidencia (MBE) y que se resumen en la figura 1. La primera etapa, consiste en formular una pregunta educativa y definir el nivel de alcance de esa pregunta, a través del modelo de Kirkpatrick (Centeno \& Ramírez, 2003). Luego, se realiza la búsqueda de la mejor evidencia disponible en torno a esa inquietud, siendo esta un desafío constante, porque el lenguaje controlado y otras herramientas de búsqueda en educación son diferentes al empleado en la investigación clínica (Haig, 2003; Rivera \& Letelier, 2007). La tercera etapa es el análisis crítico de la literatura científica encontrada y el acrónimo que se utiliza para este fin es el QUESTS: "Quality", "Utility", "Extent", "Strength", "Target" y "Setting" (Harden \& Hart, 2000). Posteriormente, la cuarta etapa es la implementación del cambio, acá se examinan las barreras y facilitadores para la ejecución de este cambio, desde la perspectiva institucional e individual (Rivera \& Letelier, 2007; Vidal \& Vicedo, 2017). Finalmente, la quinta etapa es la evaluación del cambio que determina si la modificación educativa contribuyó al aprendizaje de los estudiantes. También en esta fase, se incluye la nueva generación de conocimiento a partir del cambio realizado que ayudará a forjar una "cultura de la evidencia" (Rivera \& Letelier, 2007; Vidal \& Vicedo, 2017).

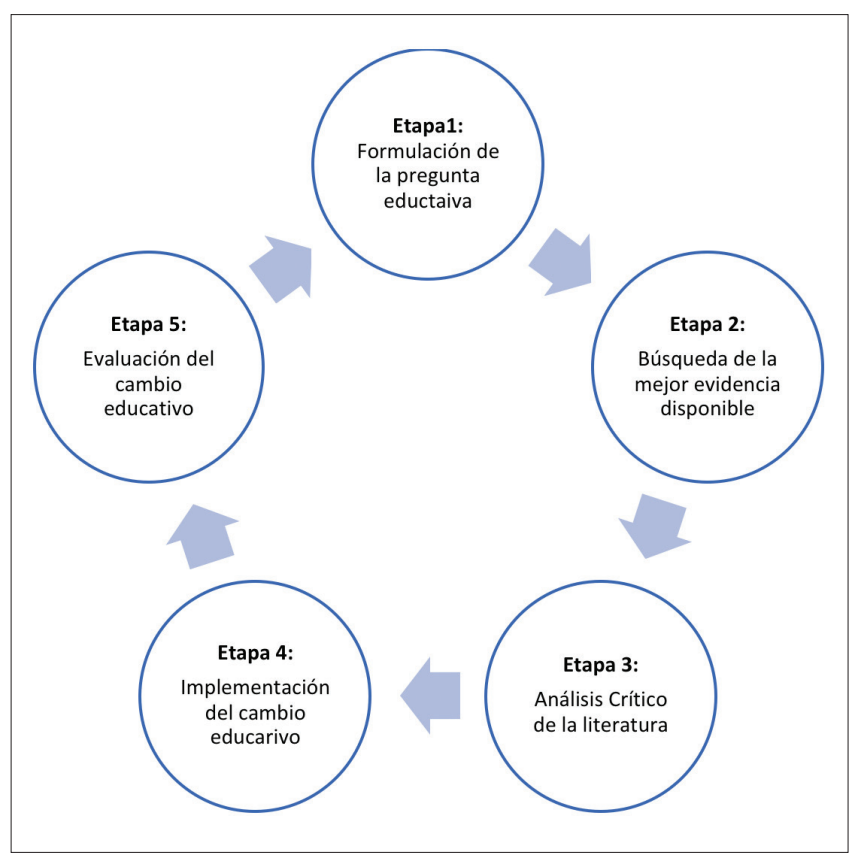

Figura 1: Etapas de la educación médica basada en evidencia.

Junto con la aparición de la colaboración BEME, surge el concepto de "educación médica basada en evidencia" (EMBE) (Harden et al., 1999; Harden \& Lilley, 2000). La EMBE es definida como "Ia implementación, por profesores en su práctica, de métodos y aproximaciones a la educación basados en la mejor evidencia disponible" (Harden et al., 1999). Esta nueva valoración de la evidencia, por parte de los profesores, lleva a efectuar un análisis de la literatura científica publicada hasta entonces. Entre los años 1996 y 2003, los estudios científicos en docencia son principalmente artículos de baja rigurosidad metodológica (descriptivos), por lo tanto, no es posible extrapolar sus resultados (Gale \& Grant, 1997; Centeno \& Ramírez, 2003; Dauphinee \& Wood-Dauphinee, 2004; Rivera \& Letelier, 2007; Cobos, 2016). En esta línea, Norman (2000) plantea que "la educación no puede ser la hermana suave de la investigación clínica". Por este motivo, que la colaboración BEME juega un rol importante en el diseño y difusión de la evidencia que sintetiza las mejores prácticas docentes (Dauphinee \& Wood-Dauphinee, 2004: Hammick, 2005; Sánchez-Mendiola, 2012; Vidal \& Vicedo, 2017). Desde el año 2005 en adelante, se convocan más congresos de educación médica a nivel internacional, existe un compromiso en financiar estudios de mayor consistencia, como son las revisiones sistemáticas, aparecen revistas especializadas en educación médica y se indexan mayor cantidad de publicaciones en este tema (Dauphinee \& Wood-Dauphinee, 2004; Hammick, 2005; Cobos, 2016; Vidal \& Vicedo, 2017). Este contexto es relevante, puesto que no sólo el docente debe avalar su práctica 
con estudios científicos, sino que también lo deben hacer las instituciones educativas (Gutiérrez \& Posada, 2004; Sánchez-Mendiola, 2012; Vidal \& Vicedo, 2017). En la medida que las instituciones integren la evidencia en el desarrollo de sus planes de estudio, se podrá enriquecer el proceso de enseñanza-aprendizaje de los estudiantes y así lograr mejorar la calidad de la atención en salud que se proporciona a las personas (Gutiérrez \& Posada, 2004).

Otro aspecto a considerar sobre la EMBE, es la dificultad que se tiene por efectuar una investigación con una "buena base metodológica", debido a que según Norman (2000), "las intervenciones en educación no son fácilmente definibles y replicables". Esta primicia refleja que la mayoría de los académicos poseen un enfoque cuantitativo muy marcado, por ende, buscan un resultado objetivo, un número (Norman, 2000; Centeno \& Ramírez, 2003). Sin embargo, en educación, muchas de las preguntas no pueden ser contestadas con la jerarquía de la investigación cuantitativa y se responden con estudios observacionales, cualitativos o mixtos (Norman, 2000; Dauphinee \& Wood-Dauphinee, 2004; Sánchez-Mendiola, 2012). Ahora, es una realidad que algunos docentes no "valoren" la investigación cualitativa, por lo mencionado anteriormente, no obstante, la investigación cualitativa es igual de "estricta" que la cuantitativa, lo que varía es el paradigma desde donde se genera la investigación y la interpretación de sus resultados (Norman, 2000; Sánchez-Mendiola, 2012). En otras profesiones no médicas, esta concepción no es igual, un ejemplo de ello es la carrera de enfermería, donde la evidencia disponible en educación, se encuentra en ambos tipos de investigación, sin embargo, el enfoque cualitativo y mixto son los que más sobresalen por la naturaleza de la profesión (Acevedo-Gamboa, 2009). Sumado a este escenario, también existe otro "desafío" en aplicar la EMBE que consiste en que muchos de los cuestionamientos del accionar docente no tienen un impacto tan claro y preciso en la salud de la población, en comparación con la investigación clínica (Murray, 2003). Esta situación se manifiesta, ya que es complejo evaluar el impacto de una intervención educativa en la salud de las personas, porque existen una multiplicidad de factores que inciden en el resultado final (Murray, 2003). Entonces, la investigación en educación puede ser "poco apreciada" e incluso apartada por algunos profesores, que deben preferir la actividad asistencial y la investigación clínica (Rivera \& Letelier, 2007). Por lo anterior, se invita a la comunidad académica a trabajar en una visión más flexible sobre la EMBE y reconocer que si bien, es importante incorporar la evidencia en la toma de decisiones en educación, también se debe sopesar estos retos y distinguir que en algunas ocasiones los estudios no responden a una lógica del paradigma cuantitativo, no obstante, tienen el mismo valor en respaldar la práctica docente.
La EMBE está creciendo paulatinamente en Latinoamérica y en otras profesiones de la salud (Cobos, 2016; Vidal \& Vicedo, 2017). En la actualidad, se ha progresado en la generación de evidencia, los docentes asisten a más congresos nacionales e internacionales en el ámbito educativo y han emergido revistas científicas en educación en varias disciplinas no médicas (Vidal \& Vicedo, 2017). A pesar de esto, aún hay profesiones de la salud, en que la introducción de la evidencia científica en la práctica docente, es más incipiente. En este sentido, es relevante que toda la comunidad educativa comience a aplicar el concepto de "educación médica y de ciencias de la salud basada en evidencia", con el objetivo de integrar la evidencia, no sólo en medicina, sino que también en las otras carreras de salud, ya que se fomentará el trabajo interdisciplinar y los estudiantes de todas las carreras de la salud se beneficiarán de esta aproximación en su formación profesional.

\section{Desafíos actuales y futuros de la educación médica y de ciencias de la salud basada en evidencia}

Antes del inicio de la pandemia por COVID-19, la educación médica y de ciencias de la salud basada en evidencia, se posicionaba fuertemente en las instituciones educativas, con un crecimiento exponencial en publicaciones, realización de congresos anuales internacionales y nacionales en educación y con una mayor cantidad de evidencia más rigurosa desde la perspectiva metodológica. Sin embargo, la pandemia por COVID-19 que comienza en el año 2020 y que sigue en la actualidad, ha impactado al mundo entero y en este sentido, la educación también ha sido desafiada (Gordon et al., 2020; Núñez-Cortés et al., 2020; Vergara et al., 2020; Daniel et al., 2021). Lo cierto es que esta pandemia ha obligado a los docentes a "actuar sobre la marcha" en varias cuestiones que aún no estaban del todo resueltas (Gordon et al., 2020; Núñez-Cortés et al., 2020; Vergara et al., 2020; Daniel et al., 2021). Por este motivo, los grandes desafíos que han sorteado las comunidades académicas se produjeron por las restricciones en la movilidad, donde muchos países implementaron medidas que favorecían el aislamiento social, por ende, se limitó el acceso a los centros educacionales y centros de salud de todas las carreras (Núñez-Cortés et al., 2020; Vergara et al., 2020). Esta situación, trasformó la modalidad en que se estaba efectuando el proceso educativo, donde se migró de una modalidad presencial a una educación en línea de emergencia, por lo tanto, los profesores comienzan a cuestionarse sobre el aprendizaje de los estudiantes en estas circunstancias (Durak \& Çankaya, 2020; Gordon et al., 2020; Núñez-Cortés et al., 2020; Vergara et al., 2020; Daniel et al., 2021). Hay que considerar que la implementación de una educación en línea de emergencia, conlleva costos que no son menores y que dependerán del desarrollo de cada país, por ejemplo, en Latinoamérica existen países que no tienen una buena conexión a internet, no tienen un completo acceso a computadores, lo que condiciona el aprendizaje 
de esos estudiantes (Durak G \& Çankaya S, 2020; Núñez-Cortés et al., 2020; Vergara et al., 2020). Lo cierto es que la educación médica y de ciencias de la salud basada en evidencia, tuvo que dar respuesta a esta interrogante y en un esfuerzo enorme muchos docentes generaron evidencia de sus experiencias particulares y difundieron las mejores prácticas que en un contexto de incertidumbre apoyaron la toma de decisiones (Gordon et al., 2020; Núñez-Cortés et al., 2020; Vergara et al., 2020; Daniel et al., 2021).

Es así como, los desafíos futuros de la educación médica y de ciencias de la salud basadas en evidencia se centran en reunir, sintetizar y difundir la evidencia que se ha construido en este tiempo, dado a que la pandemia por COVID-19 seguirá (Daniel et al., 2021). Por consiguiente, será clave descubrir que intervenciones educativas fueron efectivas para el aprendizaje y cuales no lo fueron, para así mejorar la formación de los profesionales de la salud en este período. También, hay que reconocer que la "evidencia" puede estar presentada con distintos paradigmas y por ello, a la hora de analizar críticamente un estudio se debe tener una visión más flexible.

Por último, el desafío que queda es lograr la integración del enfoque basado en evidencia en todas las carreras de la salud, ya que cada docente es responsable de formar a los mejores profesionales en virtud de la salud de las personas. Por ello, el trabajo colaborativo e interdisciplinar ayudará a llegar a esta meta.

\section{Conclusiones}

La educación médica y de ciencias de la salud basada en evidencia presenta varios desafíos futuros, ya que este es un movimiento que debe continuar con su consolidación, crecer en aquellas carreras de la salud que aún no han adoptado del todo esta concepción y adquirir una visión más flexible a la hora de analizar la evidencia disponible. También se debe considerar que la pandemia por COVID-19, ha puesto a prueba a la comunidad educativa en todas las áreas. Este hecho ha fortalecido la toma de decisiones educativas basadas en evidencia, debido a que las redes internacionales y nacionales presentan una mayor apertura, con el objetivo de generar un trabajo colaborativo que ayude a formar los mejores profesionales de la salud en todo el mundo. En conclusión, la educación médica y de ciencias de la salud basada en evidencia es y será el mejor fundamento de la práctica docente y este alcance se debe integrar en todas las instituciones educativas, con el propósito de brindar una atención de salud de calidad a las personas.

\section{Reconocimientos}

Sin financiamiento. Carolina Guerra autor principal del manuscrito y Paola Carrasco revisor del manuscrito. Sin conflictos de interés ambos autores.

\section{Referencias}

Acevedo-Gamboa F. (2009). Construcción metodológica para la enseñanza de la disciplina de enfermería: aspectos históricos y reflexiones. Investigación en Enfermería: Imagen y Desarrollo 11, 53-66.

Centeno A. \& Martínez Carretero J. (2003). Innovaciones, investigación y evidencias en educación médica: La colaboración EMBE (Educación Médica Basada en la mejor Evidencia). Educación Médica 6, 32-36.

Cobos H. (2016). Lectura crítica de investigación en educación médica. Investigación en Educación Médica 5, 115-120.

Daniel M, Gordon M, Patricio M, Hider A, Pawlik C, Bhagdev R, Ahmad S, Alston S, Park S, Pawlikowska T, Rees E, Doyle AJ, Pammi M, Thammasitboon S, Haas M, Peterson W, Lew M, Khamees D, Spadafore M, Clarke N, et al. (2021). An update on developments in medical education in response to the COVID-19 pandemic: A BEME scoping review: BEME Guide No. 64. Medical Teacher 43, 253-271.

Dauphinee WD. \&Wood-Dauphinee S. (2004). The need for evidence in medical education: the development of best evidence medical education as an opportunity to inform, guide, and sustain medical education research. Academic Medicine: journal of the Association of American Medical Colleges 79, 925-930.

Durak G. \& Çankaya S. (2020). Emergency Distance Education Process from the Perspectives of Academicians. Asian Journal of Distance Education, 15, 159-174.

Gale R. \& Grant J. (1997). AMEE Education Guide No 10: Managing change in a medical education context: guidelines for action. Medical Teacher 19, 239-249.

Gordon M, Patricio M, Horne L, Muston A, Alston SR, Pammi M, Thammasitboon S, Park S, Pawlikowska T, Rees EL, Doyle AJ. \& Daniel M. (2020). Developments in medical education in response to the COVID-19 pandemic: A rapid BEME systematic review: BEME Guide No. 63. Medical Teacher 42, 1202-1215.

Gutiérrez Rodas JA. \& Posada Saldarriaga R. (2004). Tendencias mundiales en educación médica. latreia 17, 130-138.

Haig A. \& Dozier M. (2003). BEME Guide no 3: systematic searching for evidence in medical education-Part 1: Sources of information. Medical Teacher 25, 352-363.

Haig A. \& Dozier M. (2003). BEME guide no. 3: systematic searching for evidence in medical education-part 2: constructing searches. Medical Teacher 25, 463-484. 
Hammick M. (2005). A BEME review: a little illumination. Medical teacher 27, 1-3.

Harden RM, Sowden S. \& Dunn WR. (1984). Educational strategies in curriculum development: the SPICES model. Medical Teacher 18, 284-297.

Harden RM, Grant J, Buckley G. \& Hart I. (1999). BEME Guide No. 1: Best Evidence Medical Education. Medical Teacher 21, 553-562.

Harden RM. \& Lilley PM. (2000). Best evidence medical education: the simple truth. Medical Teacher 22, 117-119.

Hart I \& Harden RM. (2000). Best evidence medical education (BEME): a plan for action, Medical Teacher 22, 131-135.

Murray E. (2002). Challenges in educational research. Medical Teacher 36, 110-112.

Norman G. (2000). Reflections on BEME. Medical Teacher 22, 141-144.

Núnez-Cortés JM, Reusi R, García M. \& Falasco S. (2020). COVID-19y la educación médica, una mirada hacia el futuro. Foro lberoamericano de Educación Médica (FIAEM). Educación Médica 21, 251-258.
Petersen S. (1999). Time for evidence based medical education. BMJ (Clinical research ed.) 318, 1223-1224.

Rivera S \& Letelier M. (2007). Analizando la evidencia en educación médica ¿se puede hacer Educación Médica basada en Evidencia?. ARS MEDICA Revista de Ciencias Médicas 36, 26.

Sánchez-Mendiola, M. (2012). Educación médica basada en evidencias: ¿Ser o no ser?. Investigación en Educación Médica 1, 82-89.

Thistlethwaite J. \& Hammick M. (2010). The Best Evidence Medical Education (BEME) Collaboration: Into the next decade. Medical Teacher 32, 880-882.

Van der Vleuten CPM, Dolmans DHJM \& Scherpbier AJJA. (2000). The need for evidence in education. Medical Teacher 22, 246-250.

Vergara de la Rosa E, Vergara T, Álvarez M, Camacho L. \& Gálvez J. (2020). Educación médica a distancia en tiempos de COVID-19. Educación Médica Superior 34, e2383.

Vidal M. \& Vicedo A. (2017). Educación médica basada en la mejor evidencia. Educación Médica Superior 31, 244-253. 